\title{
Study on the fluid flow model of fractured low permeability reservoir
}

\author{
Mingyang WU ${ }^{1, a}$, Jianjun LIU ${ }^{1,2, b}$ and Yichen LIU ${ }^{1, c}$ \\ ${ }^{1}$ School of Geoscience and Technology, Southwest Petroleum University, Chengdu China \\ ${ }^{2}$ State Key Laboratory of Oil and Gas Reservoir Geology and Exploitation, Southwest Petroleum \\ University, Chengdu China
}

a603364975@qq.com, b315245690@qq.com, c 839127124@qq.com

\begin{abstract}
Keywords: Low permeability; Fracture; Mathematical model; Seepage; Numerical simulation Abstract. Low permeability reservoir often have a variety of scales, distribution of complex natural fractures, using hydraulic fracturing which would create some large scale artificial fracture, in order to abstain more accurately prediction and judge oil production, the research on the mathematical model of fractured low permeability reservoir is particularly important. This paper has introduced the basic principle of the mathematical model of fluid flow in the reservoir of low permeability fractured proposed by scholars at home and abroad, and has carried on the analysis of the advantages and disadvantages of the mathematical model.The paper has given us some advices and pointed out the direction of development in the current and future technology of fractured low permeability reservoir,it has a signinficant effect on future fractured low permeability reservoir model research.
\end{abstract}

\section{Introduction}

Low permeability reservoirs reservoirs through history refers to the deposition, reservoir dense, low porosity, small throats, poor fluid permeability reservoirs, usually require the transformation of the reservoir in order to maintain normal production of oil and gas fields. Due to construction at the reservoir of natural fracture appeared in varying degrees of development, small-scale micro-cracks, the cracks visible scale, large-scale formation fracture. Low permeability reservoirs in the distribution of complex fractures, partial cracks, and the cracks in the main angle high angle, showing permeability anisotropy. Currently widely used in the hydraulic fracturing of low permeability reservoirs mining, hydraulic fracturing promote the generation of large-scale reservoir cracks, and deepen the local distribution of low permeability reservoirs cracks. In order to accurately predict the recovery of low permeability reservoirs, scholars reservoir fracture fluid flow model has been a lot of research work proposed dual media model, equivalent continuum model, discrete medium model, triple media models model.

\section{Percolation Model of Low Permeability Oil Reservoir}

(1) Dual Continuum Model

Warren and Root (1963), Kazemi (1969), and De Swaan (1971) established the reservoirs is mainly suitable for complete fracture and communicate with each other, used to simulate the flow of low permeability reservoirs early double medium model. In order to simulate the actual fractured low permeability reservoir reservoir fluid flow, Warran and Root (1963) simplified the fractured reservoir is complex orthogonal grid system, Kazemi and DeSwaan further study, so as to form an early double medium model. Dual media model the fracture and reservoir matrix as intertwined media considered, the presence of each of these two media of different permeability, fluid flow in the fracture and reservoir matrix is relatively independent, and between them substance traffic exchange took place. Medium flowing fluid causes a change in permeability reservoirs, changes in permeability and affects the fluid flow in porous media, this fluid interaction between the flow and deformation of the medium called flow - solid coupling.

Mainly at home and abroad to study the flow of fractured low permeability reservoir fluids double medium model. Dual continuum model there are two holes with two holes and single permeability model dual permeability model. 
Infiltration model and single with two holes with two holes dual permeability model with dual medium model as the reservoir medium regarded as a two-part system consisting of bedrock and fracture system in the actual petroleum engineering, the system also includes a rock pores, holes, caves and other medium and large cracks in the reservoir fracture system by micro-cracks and tectonic produce large cracks and artificial components. They differ, double single permeability model assumes that only the reservoir fluid to flow through the wellbore fracture system porosity bedrock within the system, holes, caves and other media only as a reservoir storage space. The dual permeability model assumes holes reservoir fluids through the fracture system and matrix systems simultaneously flow to the wellbore. In hydraulic fracturing of low permeability reservoirs can obviously ignore bedrock system fluid flow to the well bore, large cracks artificially low permeability reservoirs constructed for the role and failed to form the bedrock of the system fluid to the wellbore circulation cannot be ignored.

Development of dual media model considers the rock matrix in the non-Darcy permeability model, mainly for fractures and interconnected reservoir, but channeling function matrix and reservoir rock is difficult to determine the crack between the coupling complex mathematical equations, not suitable for strong directional plane, cannot form the fracture network of low permeability reservoir. At the same time, when the low permeability reservoirs have many cracks in the macro-scale, there is a big double medium model error.

(2) Equivalent Continuum Model

Foreign scholars Snow (1969), Long (1982), Oda (1986) put forward the equivalent continuum model, Domestic scholars Liu Jianjun, Feng Jinde done a lot, and Liu Jianjun, etc. According to the equivalent thought, established equivalent continuum percolation model is suitable for fracture unconnected reservoirs, as shown below.

Feng Jinde and other considerations fractured low permeability reservoir fracture development characteristics of the naturally fractured reservoir parameters and combine An equivalent continuum model fractured low permeability reservoirs. For the equivalent continuum model does not apply to the development of reservoir pores, Xu Xuan, etc. using capillary bundle model, established the principle of equivalent flow resistance equivalent medium model cavern Carbonate Reservoir.

For low permeability, ultra-low permeability reservoirs, the reservoir having a distribution of complex, anisotropic crack scale variable. Therefore, in the actual reservoir engineering, the penetration of the crack with a locality. Equivalent continuum model ignores local effects of natural fractures, from a macro-scale considerations anisotropy naturally fractured reservoir flow capacity caused by the equivalent permeability tensor to represent. Equivalent continuum percolation model to simulate the low permeability reservoir, lies the key to determining the equivalent permeability tensor determination, the equivalent reservoir permeability and reservoir porosity and other reservoir fluid flow-related parameters, domestic foreign scholars have considered the natural fracture distribution, the impact of connectivity and cut through the cracks extent, fracture linear density equivalent crack opening degree of permeability, and the impact of crack on the rock deformation parameter and the equivalent permeability and strain relationship. By complex flow pattern is equivalent to simple flow law and discontinuous geological model is equivalent to a continuous geological model, researchers using the equivalent continuum model will have a variety of joints anisotropic low permeability reservoir with each reservoir rock to a uniform description (see below).

Establishment of equivalent continuum model there are several key steps: (1) to medium region under study meshing; (2) calculable each grid block containing the fractured structure, etc. permeability tensor effect; (3) the homogeneous anisotropic continuum body characterized by the equivalent permeability tensor; (4) after characterization of the resulting homogeneous anisotropic continuum mention instead of the original heterogeneous discrete medium body, a new combination of equivalent study area; (5) and then use the fully anisotropic permeability tensor full continuum percolation theory analysis; (6) the model with the appropriate boundary conditions, while balancing substance list balance equation; (7) to further solve the scale and direction to ensure that the fluid flowing from the heterogeneous and homogeneous rock rocks are the same. 
Equivalent continuum models from macro to consider large-scale use of the equivalence principle anisotropic low permeability reservoirs regarded as isotropic homogeneous rock, using a very fine mesh and cracks near the reservoir matrix, its advantage is that the model is simple, mature theory, can accurately describe the pressure and phase saturation within the fracture fluid, and the quality of exchange between the fracture and the matrix. But the model is difficult to determine the equivalent permeability coefficient.

(3) Discrete Medium Model

Cracks in low permeability reservoirs have heterogeneity, reservoir fracture scale differences, and distribution of crack and complex reservoirs should belong to the class of discrete media. Consider the reservoir fracture distribution of local features have, to a more realistic description of fractured low permeability reservoirs in fluid flow, at home and abroad singlet media model to make improvements, taking into account the degree of opening of the reservoir fracture away less than the calculated grid scale that can be ignored on the inner crack Jian direction of physical changes in the numerical simulation of fracture geometry grid on the proposed merger discrete medium model [30-33]. Chinese scholars consider the multiscale effect shale gas development in the seepage is proposed based on a crack - discrete fracture model dual porosity media. In this model, the matrix, natural and artificial fracture using their equations calculated independently, between different media associated with each other through the exchange of traffic.

Equivalent permeability is a critical parameter fracture numerical simulation of fluid flow, Yan $\mathrm{Xia}$ and other considerations of each space crack distribution and properties of the flow parameters on the establishment of a new method for calculating the equivalent permeability fractured media. According to the model of discrete media, scholars and the development of discrete fracture model, embedded discrete fracture model, discrete fractured network model as applied to models with different reservoir research.

Discrete medium model itself is based on the discrete reservoir properties of the medium evolved to highlight the characterization of single hydraulic fracture characteristics, thereby accurately depict the fluid flow in fractured rock mass. For the use of hydraulic fracturing forming a plurality of artificial reservoirs and large cracks, seepage effect only from the macroscopic scale to consider more suitable large cracks, and cracks in the actual reservoirs and caves high number, distribution complex, the model computationally expensive. At the same time, the actual distribution of the reservoir contained cracks and caverns of varied discrete medium model need to gather more accurate information on the distribution of cracks in the cave, therefore, to establish a real reservoir fracture cave quite difficult to achieve a network system.

(4) Triple Medium Model

For a more realistic description of low permeability reservoirs in fluid flow, foreign scholars Clossman according to the actual situation of the reservoirs proposed triple medium model. China is proposed by Wu and Ge Li Yushu first, Zhang Dongli and other scholars of the fluid-solid coupling numerical model of triple media model a lot of research. Then Liu Ciqun, Yao Jun, Wang Zisheng etc. according to the characteristics of low permeability reservoirs will be further developed triple medium model, the fractured media type considered by the high permeability fractured, low permeability rock and cave composed by channeling the quasi steady state stream function link. Jiangchun Tang established a three-phase triple-medium model for hydraulic fracturing capacity to predict. Zhu Qin will be considered by the artificial medium large triple fracture network, natural fracture network and micro-rock matrix composition, the establishment of a new triple-media model. Vuggy low permeability reservoirs triple media model as shown below.

Triple medium fracture - cavity model currently in low permeability reservoirs is widely applied, it increases the cavity medium of the system concept, the reservoirs are divided into three different continuous media, and three media overlap in space and due to the different flow characteristics of each medium, which both independent and interrelated. With respect to the double medium can describe more accurately the actual situation of reservoirs, but it adds a dimension on the basis of double medium model, the reservoir is considered continuous medium, ignoring the distribution of reservoir fracture and cave irregularity. 


\section{Conclusions}

Fractured low permeability reservoirs complex structure, in particular when the reservoir numerical simulation model, the need for the reservoir characteristics within the matrix, cracks, caves etc. and distribution of preliminary understanding, the reservoir according to the actual situation, the establishment of closer to the mathematical model of the actual reservoir.

Double medium model is applicable to the point of view of fracture and reservoir interconnected whole, and for, a number of large-scale reservoir from the local crack in terms of time, roughly the fracture and matrix both media overlap processing, ignored local effects of large-scale fracture model errors.

Equivalent continuum model is considered a macro scale, by dividing the flow parameters of each grid will be uneven reservoirs represent a homogeneous rock, but it is difficult to determine the equivalent permeability and other parameters. Research on the model of the individual flow parameters and their influencing factors are the focus of research model for further development.

Discrete medium model, in theory, be able to simulate real reservoir in fluid flow, but due to the exploration and development of cracks limitations of computer technology, the established model is difficult to really simulate the real fluid flow. With the development of exploration technology and computer technology, discrete media model will be able to more realistically simulate real reservoir, the model will gradually become fractured low permeability reservoir simulation hotspot.

Triple double medium model medium fracture system is divided into micro-fracture system and large artificial fracture system. The low permeability reservoirs fracture treated as multiple media description, can crack multiscale characterization out preliminary reflect fractured low permeability reservoir multiscale modeling. The cracks in multi-scale modeling and multi-media model combination of different scales fracture fluid flow, and then create multiple media reservoir simulation model of production capacity, is fractured low permeability reservoir in the current technical conditions of development.

\section{References}

[1] J.J. Liu, X.G. Liu, Y.J. Ji, R. Song: Investigation of Fluid Flow in Fractured Low Permeability Oil Reservoir Coupled with Geostress (ST Plum-Blossom Press, Australia 2014).

[2] G.H. Pei, F. Yu, J.J. Liu. Journal of Residuals Science \& Technology. Vol. 12(2015), p.S85

[3] J.J. Liu, R. Song, M.M. Cui. Journal of Hydrodynamics. Vol. 27(2015), p. 234

[4] J.J. Liu, R. Song. Progress in Computational Fluid Dynamics, An International Journal. Vol. 15(2015), p.317

[5] Qin D. H., Liu L. L., Mao T., Liu J.J., Liu F.Z.. Materialwissenschaft Und Werkstoftechnik, Vol.46(2015), P. 978

[6] J.J. Liu, L.F. Zeng, Y.J. Ji, J.T. Gao. Mechanical Mechanism and Numerical Simulation of Casing Damage in Thermal Production Oilfield. (Science Press, China 2013)

[7] J.J. Liu, S.L. Chen, Y.J. Ji, G.H. Pei and Q.S. Li. Mechanism of Leakage and Interporosity Flow of Injected Fluid in Low Permeability Oil Reservoir. (Science Press, China 2015)

[8] Liu J.J., Yu X.B., H. Xiong, L.Z. Zhang. Science \& Technology Review, Vol. 31(2013), p.43 